\title{
Erratum
}

\section{A New Short Proof of the Local Index Formula and Some of Its Applications}

\section{Raphaël Ponge}

Department of Mathematics, Ohio State University, Columbus, OH 43210-1174, USA.

E-mail: ponge@math.ohio-state.edu

Received: 19 January 2004 / Accepted: 18 March 2004

Erratum published online: 18 June 2004 - (C) Springer-Verlag 2004

Commun. Math. Phys. 241, 215-234 (2003)

Unfortunately several misprints have appeared in the article A new short proof of the local index formula and some of its applications. We give a list of corrections:

- Page 216: In the line between the equations (1) and (2) the reader should read " $C^{0}\left(\mathbb{R}, L^{2}(M, \mathcal{E})\right) \subset \mathcal{D}^{\prime}(M \times \mathbb{R}, \mathcal{E})$ " instead of " $C^{0}\left(\mathbb{R}, L^{2}(M, \mathcal{E})\right) \subset \mathcal{D}(M \times$ $\mathbb{R}, \mathcal{E})$ '.

- Page 218: In Proposition 1 the $5^{\text {th }}$ line should be removed.

- Page 219: Equation (10) in Theorem 2 should read

$$
k_{t}(x, x) \sim_{t \rightarrow 0^{+}} t^{\frac{-n}{2}} \sum_{l \geq 0} t^{l} a_{l}(\Delta)(x), \quad a_{l}(\Delta)(x)=\check{q}_{-2-2 l}(x, 0,1) .
$$

Furthermore, in Proposition 2 the following changes need to be made:

i. In the first sentence: "For $t>0$ we let $h_{t}(x, y)$ denote the distribution kernel of $P e^{-t \Delta "}$ (not "of $P$ "):

ii. Equation (12), and the text which follows should read:

$$
h_{t}(x, x) \sim_{t \rightarrow 0^{+}} t^{\left[\frac{m}{2}\right]-\frac{n}{2}} \sum_{l \geq 0} t^{l} b_{l}(x), \quad b_{l}(x)=\check{r}_{2\left[\frac{m}{2}\right]-2-2 l}(x, 0,1),
$$

where the equality on the right-hand side gives a formula for computing the densities $b_{l}(x)$ 's in local trivializing coordinates using the symbol $q \sim \sum q_{m-2-j}$ of $P\left(\Delta+\partial_{t}\right)^{-1}$ (or of $P Q$ where $Q$ is any Volterra parametrix for $\Delta+\partial_{t}$ ).

- Page 234: The correct reference for Richard Melrose's book is:

\section{References}

[Me] Melrose, R.: The Atiyah-Patodi-Singer index theorem. Boston: A.K. Peters, 1993 